\title{
Seismogram Analysis of the Earthquakes in Sumatra on WRAB Observation Station: S Wave Velocity Structure on Subduction Zone of Sumatra-Java.
}

\author{
Bagus Jaya Santosa ${ }^{1}$ \\ ${ }^{1}$ Program Studi Geofisika, FMIPA \\ Institut Teknologi Sepuluh November, Surabaya
}

\begin{abstract}
The S wave velocity structure at subduction zone under Sumatra-Java was investigated through seismogram analysis in time domain and three Cartesian's components simultaneously. The main data set was the comparison between the measured seismogram and the synthetic one, not the travel time data. The synthetic seismogram was calculated with the GEMINI method. The seismogram comparison shows that the global earth mantle of PREMAN gives deviating synthetic seismogram and has later arrival times than the measured one. The gradient $\beta_{\mathrm{h}}$ in the upper mantle is altered to positive from its negative slope as in the PREMAN model, and positive corrections are added to the zero order of polynomial's coefficients in all earth mantle layers. The excellent fitting, as well as travel time or waveform, were obtained on the surface waves of Love and Rayleigh, the S and SS mantle and repetitive depth waves. The additional positive corrections were also confirmed by a well fitting on the repetitive depth waves. This result expresses that part of the earth mantle that due to tectonic processes has positive anomaly on $\mathrm{S}$ wave velocity and vertical anisotropy in all of the earth mantle layers.
\end{abstract}

Keywords: positive anomaly; seismogram analysis; subduction zone; vertical anisotropy.

\section{$1 \quad$ Introduction}

Since the technological advances the investigation in the earth structure has constituted a challenging subject and has given a vital information to understand the global tectonics and the dynamic evolution of our earth. For the last two decades, by exploiting the computer capacity and the improvement of the seismic data quality, seismologists have opened a new field of seismology, that is the seismic tomography, and have applied this new technology to map the three dimensional structure of the earth [1-8]. For example, the forward waveform modeling has completed the tomography study giving details about the structural features in the same vein with the small-heterogeneity (refers to the last reviews $[9,10])$. The studies have developed our understanding about the structure and dynamics of the interior of the earth. The subducted

Received December $12^{\text {th }}$, 2006, Revised January 31 ${ }^{\text {st }}$, 2007, Accepted for publication January $31^{\text {st }}, 2007$. 
lithosphere layers and the expanding marine-mantle constitute two features that complement each other in the convection of the mantle [11].

The earlier global tomography study analyzes the earth structures by using blocks $[2,5]$ or the expansion of spherical harmonic function $[12,13]$. In the block models, the medium used is divided into cubical boxes, the seismic velocity in each block is assumed to be a constant, and the velocity changes quickly from one block to another. The artificial velocity interface is introduced in the block model. The spherical harmonic has difficulty to describe the subducted plates or plume. With the grid approach, the 3-D grid framework in the whole mantle is spread out first. The velocity perturbation begins with the 1$\mathrm{D}$ velocity obtained by the dispersion curve analysis. The velocity perturbation on any point in this model is calculated through the linear interpolation on adjacent eight points of cube. Therefore, in the grid approach, there are no artificial velocity changes like in the block model. The Moho depth ranges from about $10 \mathrm{~km}$ under the ocean to 40-70 km under the continent plate [14] can be spread out well in the grid model. The discontinuity of 410 and $660 \mathrm{~km}$ experiences a depth variation up to $18 \mathrm{~km}$ on the global scale [15]. A change into such discontinuity influences the wave travel time and the passage of the seismic wave beam, and therefore, this topography should be taken into account in the tomography inversion.

In the tomography studies for the mantle structure with the previous traveling time data, few seismologists have their own accurately-measured travel time data (e.g. $[13,3])$, and most of seismologists have used a large amount of the ISC (International Seismic catalogue) data. A reprocessing of data [16] has resulted in a global image about the mantle structure from the times of the Pwave from ISC, similar to the model mantle structure that is obtained from the non ISC data of the arrival time [3].

In this research, the seismogram data of the earthquakes in Sumatra that are recorded by the WRAB observation station, North Australia has been analyzed. The data contain the ray path from the earthquakes hypocenters to the observation station that passes the subduction structure in the South of Sumatra and Java. Additionally, the analyzed data set in this research was the waveform comparison in the seismogram, where the overall points of the seismogram data were analyzed. The waveform used was from $\mathrm{S}$ wave, the surface-waves of Love and Rayleigh in three Cartesian components simultaneously, and the ScS depth phases and their repetition. The travel time data [1-8] takes just some points of data in the time series of the seismogram, i.e. the arrival times of certain wave-phases in the station. The seismogram used by measuring the arrival times is generally just on the canal of Z-component. 
The ray paths from the hypocenter of the earthquakes in Sumatra-Java pass the structure of the mantle of the earth from the upper mantle to CMB (Core Mantle Boundary), where the perturbation media is the subduction structure. The earlier seismology researches $[3,4,8]$ used the arrival time data of the waves $\mathrm{P}, \mathrm{S}$ and ScS and also the dispersion analysis on the surface waves separately.

\section{$2 \quad$ Methodology}

The seismogram data were downloaded from Databank Center IRIS (International Research Institutions for Seismology). The positions of the earthquake sources are in Sumatra, Indonesia. Table 1 presents the earthquakes in this research that are recorded in the WRAB station.

Tabel 1 The Codes and positions of the earthquakes in Sumatra that are analyzed in this research.

\begin{tabular}{cccc}
\hline No & Earthquake's Code & Latitude & Longitude \\
\hline 1 & C081098A & 7,32 & 94,31 \\
2 & C103194B & 3,03 & 96,27 \\
3 & C110895A & 1,85 & 95,06 \\
4 & C081499A & $-5,89$ & 104,71 \\
5 & M060400D & $-4,72$ & 102,09 \\
6 & C021301D & $-4,68$ & 102,56 \\
7 & B020399B & $-6,19$ & 104,22 \\
\hline
\end{tabular}

The position of the WRAB observatory station, the earthquakes epicenters and vertical projection of the ray paths from the hypocenter to the observation station is presented in Figure 1.

First TTIME, a computer program to calculate the synthetic travel time of the main wave phases, needs to be run. The program was based on Bulland and Chapman's Paper [17], and was obtained from http://orfeus.knmi.nl. The synthetic travel times is used as a guide to identify the wave phase in the seismogram. To calculate the synthetic seismogram, we use a program based on GEMINI method (Green's function of the Earth by MINor Integration) [18, 19]. 


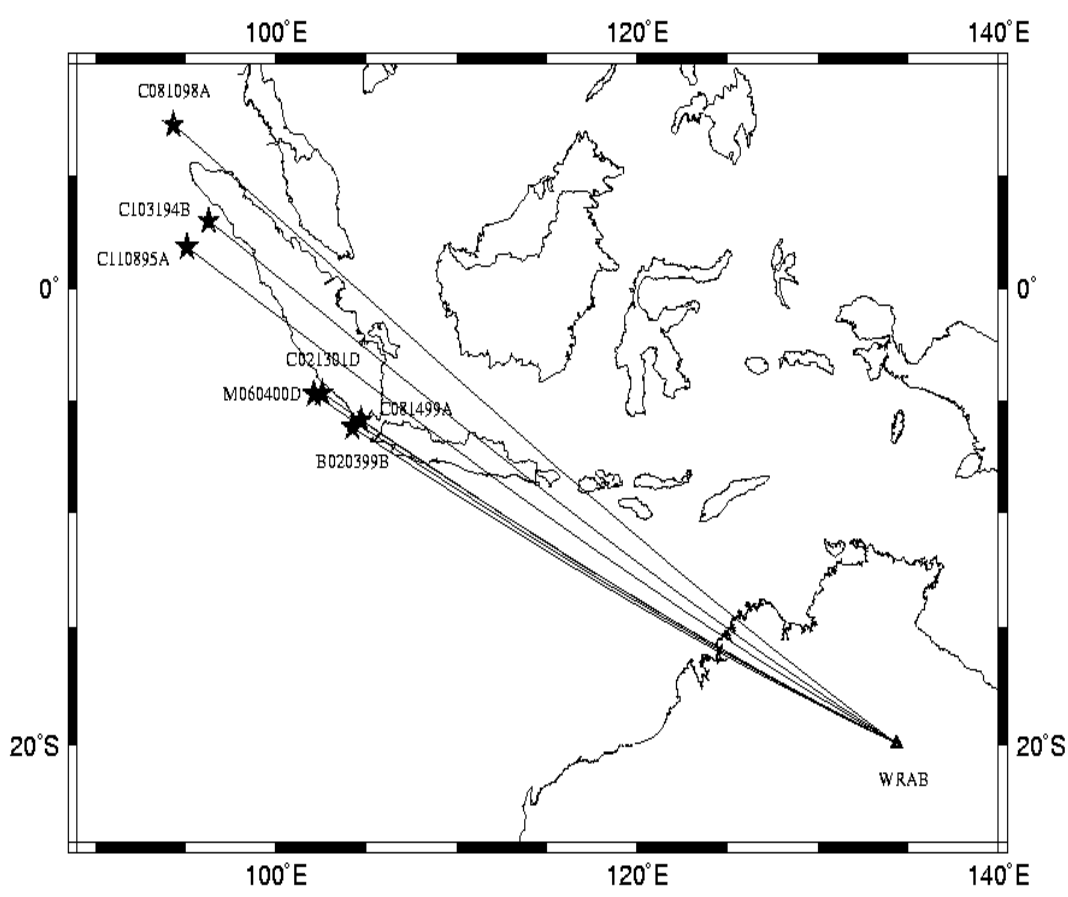

Figure 1 The vertical projection of the ray paths from the earthquake hypocenters to the WRAB observatory station.

GEMINI, stands for Green's function of the Earth by MINor Integration, is a program to calculate the minors of the Green functions for an earth model and for a certain depth of earthquake source. The green's functions are expanded (integrated) by fulfilling the physical conditions in the returning point of wave (the deepest point in wave propagation), the depth point of source and the border conditions in the earth surface. The expansion is written using the independent variable as a complex frequency by inserting a trick damping $(\omega+$ $i \sigma)$ to avoid time aliasing. The earthquake tensor moment detailed in the third line of the CMT (Centroid Moment Tensor) solution was used to calculate the coefficients of Green's function through the accomplishment of the Cramer rule on the Green's function. The right side of linear equations is the coefficient series of the tensor moments. The coordinate of earthquake source was put as the North Pole, and the coordinate of the observation station was changed into a form of epicentral and azimuth angle. The spherical harmonic function was developed with these two angle values. The DISPEC program (belongs to Gemini Package) reads the Green's function that has been produced by GEMINI program and forms a multiplication with the coefficients of expansion on the Moment Tensor and the spherical harmonic function and then sums 
them, resulting the synthetic seismogram in the complex frequency domain. The MONPR program (GEMINI Package) transforms the synthetic seismogram from the complex frequency domain into the time domain. The measured and synthetic seismograms were subjected to a Butterworth low-pass filter. The inverse RESPONSE file from the seismometer equipment system on the receiver station was imposed to the measured seismogram, i.e. the description about the phase change and the amplification of the equipment system while changing the input of the land-movement in velocity/acceleration into the output Voltage. The horizontal component of the measured seismogram should be rotated with the $\mathrm{X}$ axe (East-West canal) was directed to the small arch formed by the observation station of the earthquake source (back-azimuth), refer to Figure 1. The purpose was to decompose the wave movement in 3-D space into the components of P-SV and SH. Therefore the synthetic and measured seismograms were compared in the same unit and movement direction.

\section{$3 \quad$ Analysis and Discussion}

\subsection{Analysis}

The following figures present a comparison and fitting of seismogram on the $\mathrm{S}$, Love and Rayleigh waves to obtain the $\beta$ velocity structure on the shallow mantle layers down to the deep ones. Every figure contains three components. They are labeled with $\mathrm{Z}$ for vertical, $\mathrm{r}$ for radial, and $\mathrm{t}$ for transversal. In each component, there are three curves, in which the continuous curve is the measured data, the dots curve is the synthetic seismogram constructed from the PREMAN earth model [20], and the dots-dashes curve is the fitting seismogram obtained by using the corrected model of the earth, which is displayed on a small box on the right side as a dots-dashes curve. Generally to obtain the fitting, the velocity gradient of $\beta_{\mathrm{h}}$ at the upper mantle was changed into positive against the depth; originally it was a negative in the PREMAN earth model. Meanwhile, the gradient for $\beta_{\mathrm{v}}$ is left as PREMAN. The change was done just on the zero-order coefficients on the polynomial that describes the $\beta$ velocity function at each layer of the earth mantle.

Figure 2 presents seismogram comparison and fitting on the earthquake C081499A, Nicobar Archipelago which the data were recorded in the observation in WRAB station.

We can see that the synthetic seismogram constructed from PREMAN earthmodel provides a lagged waveform on all kinds of the S, SS, Love and Rayleigh waves. The Love wave from PREMAN also decays more quickly than the measured data. The corrected model of the earth provides a very good fitting of 
seismogram on the arrival time of oscillation in the Love wave, and also the height pattern of amplitude is more comparable to the measured data. To obtain fitting on Love waveform, the thickness of the earth crust is added $2 \mathrm{~km}$, so it becomes thicker than that in the PREMAN earth model. Fitting is also attained on the SS wave, that exists in the preliminary part of the Love wave and $\mathrm{SH}$ Wave. A good fitting is also obtained on the Ralyeigh and $S$ waves, both on $r$ and $\mathrm{z}$ components.
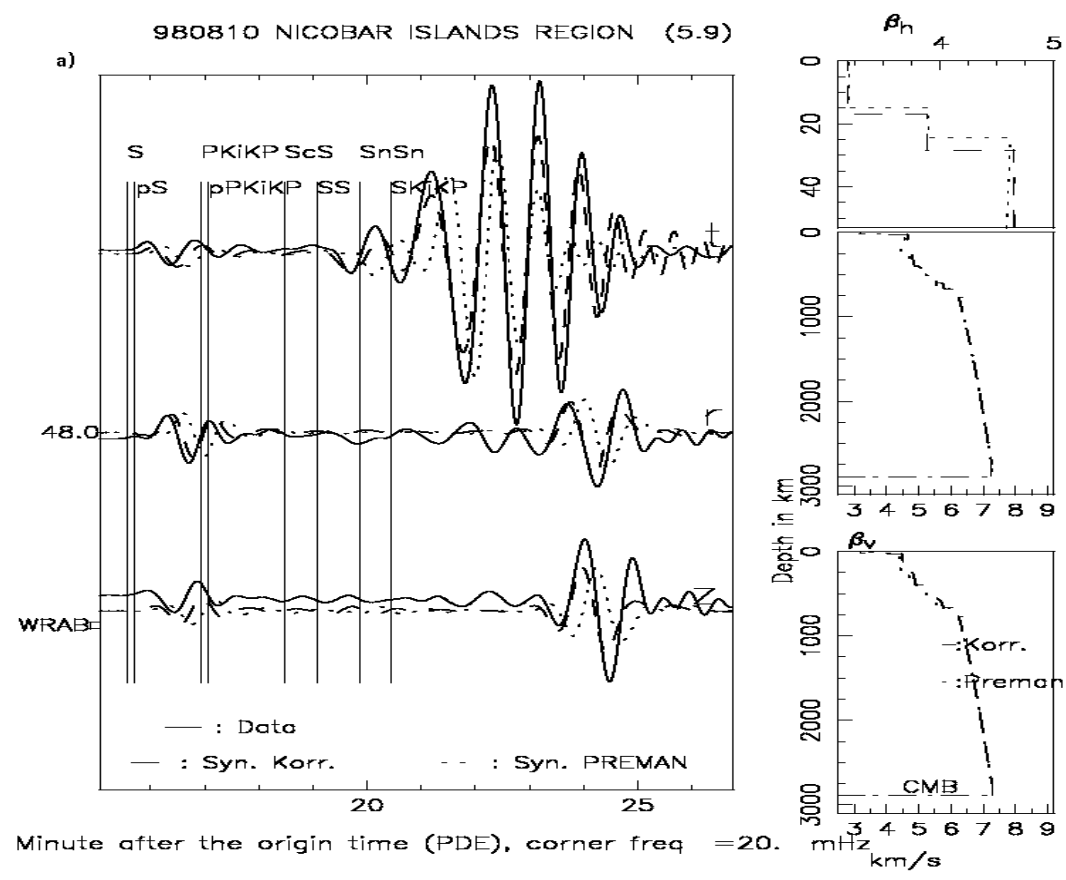

Figure 2 The seismogram comparison of the earthquake seismogram C081499A, Nicobar in WRAB station: S, Love and Rayleigh Waves.

Figure 3 presents the seismogram analysis of C103194B earthquake. The PREMAN earth model provides the lagged waveform on all kinds of waves, both Love and Rayleigh surface wave, and the shallow body wave or the repetitive depth waves $\mathrm{ScS}_{2}$ and $\mathrm{ScS}_{3}$. The corrected earth-model gives an excellent fitting, even up to the $\mathrm{ScS}_{3}$ depth wave. The excellent fitting on both depth waves $\mathrm{ScS}_{2}$ and $\mathrm{ScS}_{3}$ proves that the positive correction that has been imposed on the zero-order coefficients in the velocity polynomial function in every layer of the earth is correct. The observation of the depth waves for small epicentral-distance criticizes the results of the other seismogram research that use the data set of the arrival times PKP-P, $\mathrm{P}_{\text {diff, }}$ in which the measurement of the arrival times required the observation stations, which has a great epicentral distance [21, 22]. 

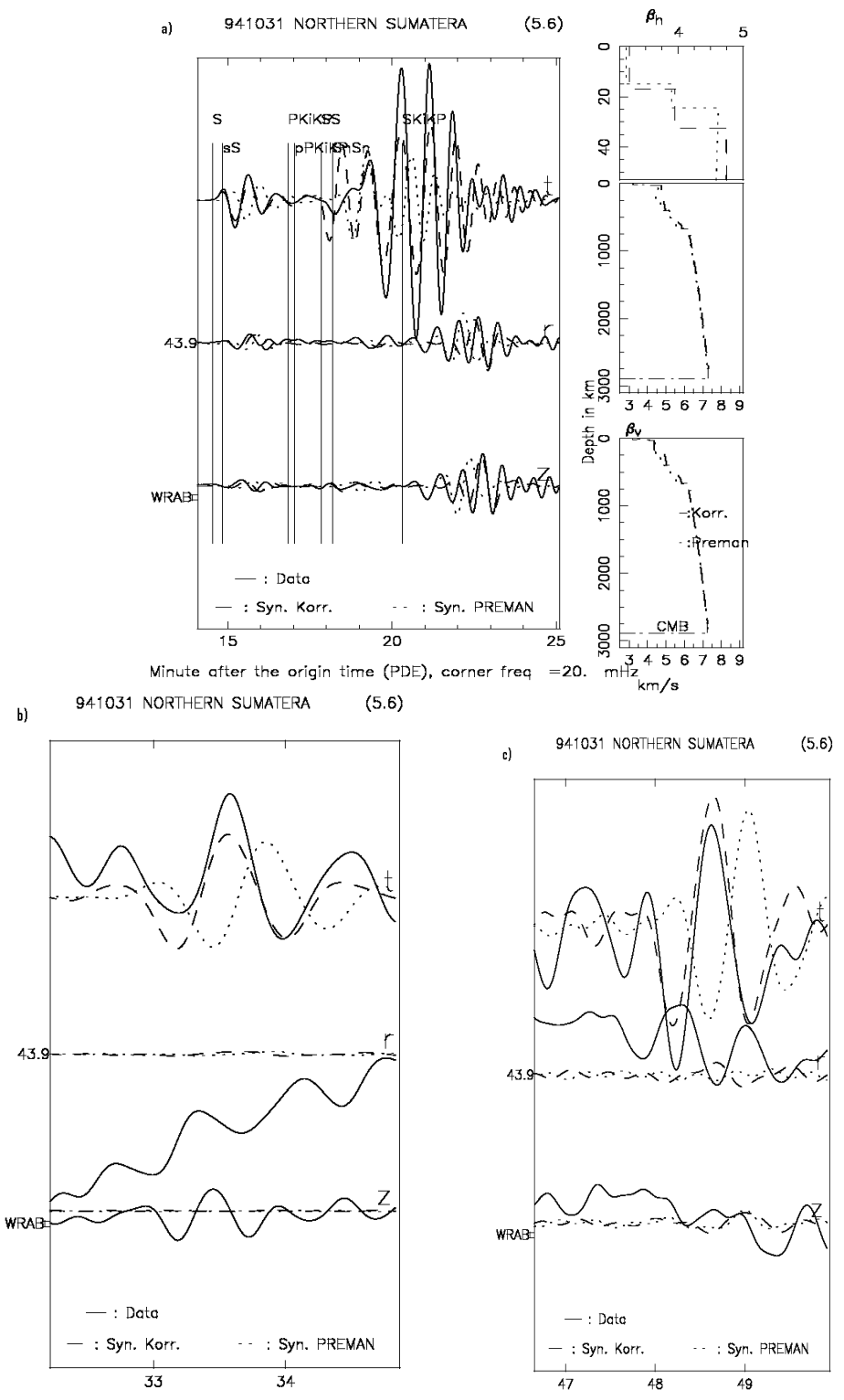

Minute after the origin time (PDE), corner freq $=20 . \mathrm{mHz}$ Minute after the origin time (PDE), corner frec $=20 . \mathrm{mHz}$

Figure 3 The seismogram comparison of the earth quake C103194B, West coast of Aceh in WRAB station: a. S, Love and Rayleigh waves; b. $\mathrm{ScS}_{2} ;$ c. $\mathrm{ScS}_{3}$.

Figure 4 shows the seismogram analysis and fitting of the earthquake C110895A that occurred on the offshore of North Sumatra. The hypocenter position of the earthquake was next to that of the previous earthquake. The analysis of the synthetic seismogram that is constructed from the PREMAN 
earth-model indicates that the synthetic waveform arrives later than the measured data on all kinds of waves, both on Love and Rayleigh surface waves, the shallow body wave or depth wave. The seismogram fitting is excellent. Interesting to note the existence of the small curve on the preliminary waveform of the Love wave in the $t$ component is very-well simulated by the synthetic waveform of the corrected earth-model. A positive correction occurs not only in the upper mantle layer, but also continues down to the mantle layers below it up to CMB. It is proved also by the attainment of fitting on the $\mathrm{ScS}_{2}$ wave, where the wave passes all the mantle layers four times.

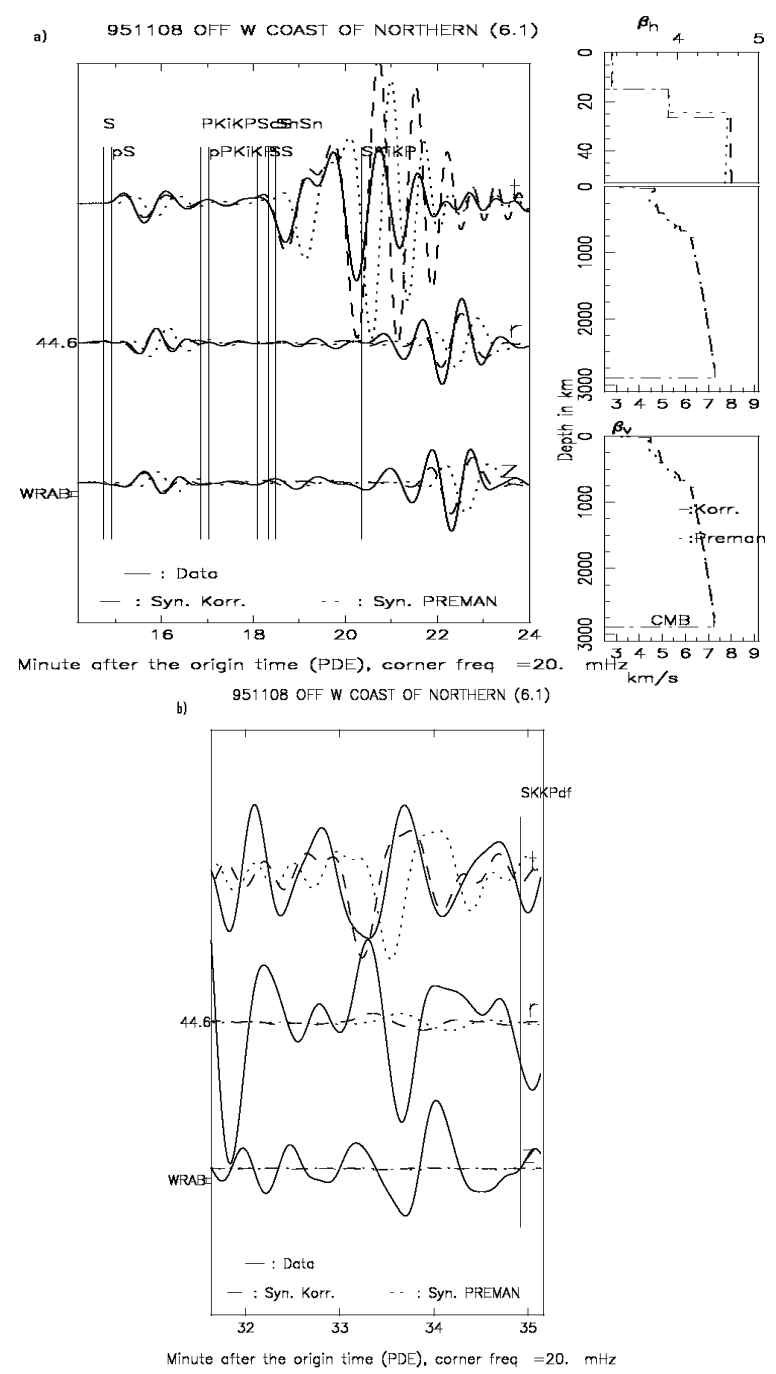

Figure 4 Seismogram comparison of the earthquake C110895A, West Coast of North Sumatra in the WRAB station: a. S, Love and Rayleigh waves; b. ScS 2 . 
Figure 5 presents a seismogram comparison of the earthquake CO21301D, South Sumatra recorded in the WRAB station. Again, the PREMAN earthmodel provides the synthetic seismogram that arrives later than the measured seismogram. By imposing the positive correction on the velocity on all of mantle layers, and also the positive gradient on $\beta_{\mathrm{h}}$ at the upper mantle, a good fitting on all kinds of $\mathrm{S}$ waves and their $\mathrm{ScS}$ repetition has been obtained.

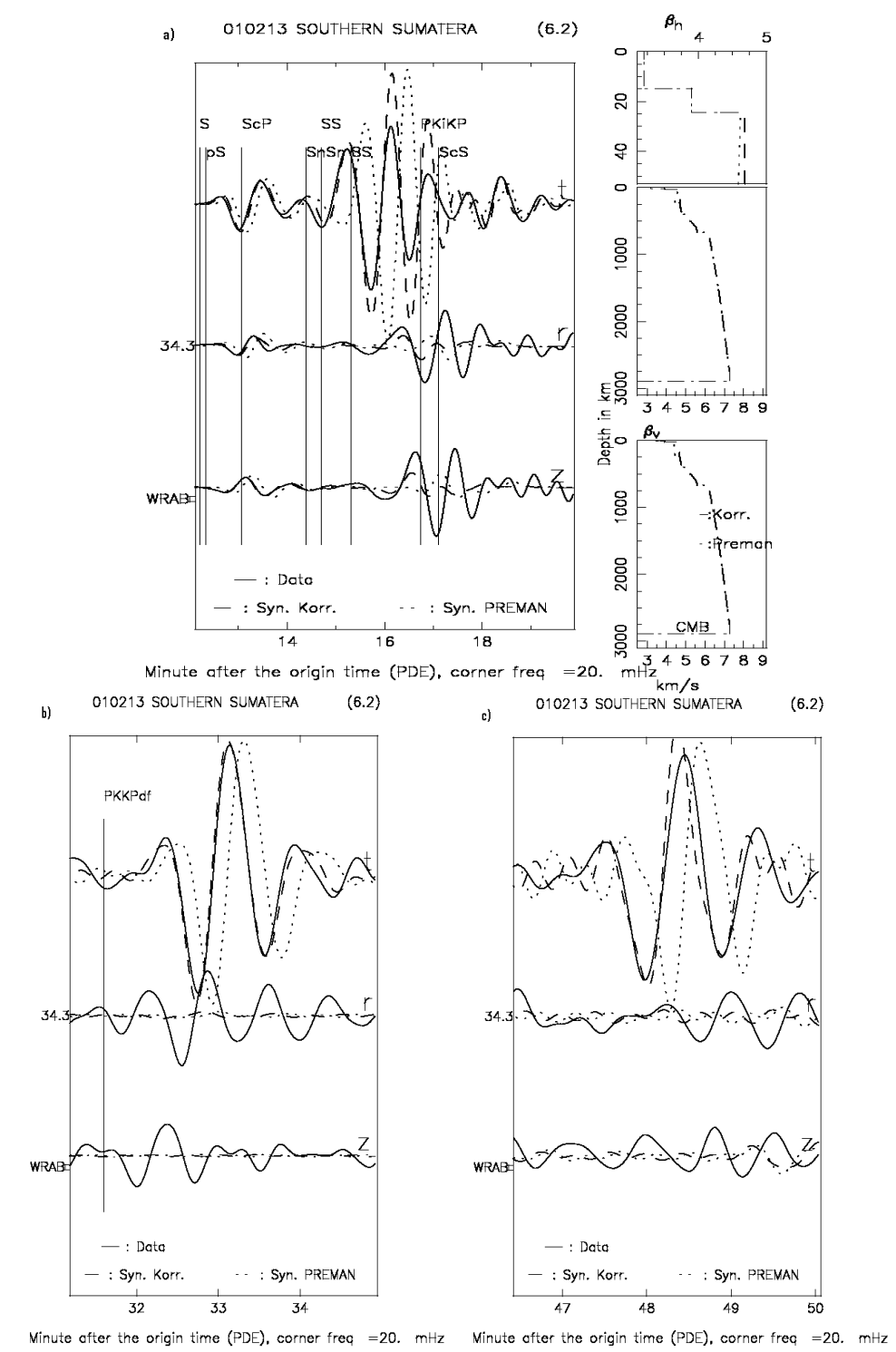

Figure 5 Seismogram comparison of the earthquake CO21301D, South Sumatra in the WRAB station: a.S, Love and Rayleigh waves; b. $\mathrm{ScS}_{2}$; c. $\mathrm{ScS}_{3}$. 


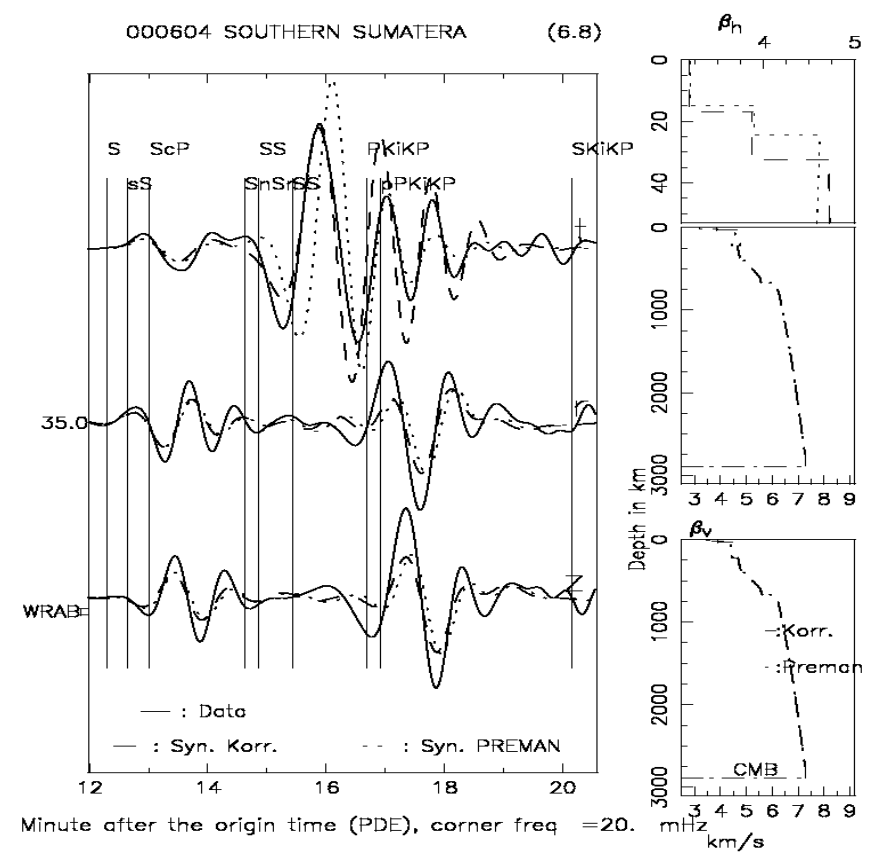

Figure 6 Seismogram comparison of the earthquake M060400D, South Sumatra in the WRAB Station: S, Love and Rayleigh waves.

Figure 6 shows the seismogram analysis and comparison of earthquake M060400D, South Sumatra in the WRAB station. The hypocenter of this earthquake is next to that of the previous earthquake. Unfortunately, the quality of this data is not good, so that only the front piece of the seismogram can be analyzed. Nevertheless on the top layer of mantle up to depth of $730 \mathrm{~km}$, a positive correction on the zero order of the velocity structure of the $S$ wave is needed, to find the seismogram fitting of the S, Love and Rayleigh waves.

Figure 7 shows the comparison of the seismogram C081499A that occurred in South Lampung, where the seismogram was recorded in the WRAB station. The epicentral distance is smaller than those of the previous earthquakes. The PREMAN earth model gives a synthetic seismogram with the arrival time behind the measured wave phases. Positive corrections are imposed on all of the mantle layers up to CMB. The magnitude of the correction is correct, because the $\mathrm{S}$, Love and Rayleigh and also the depth waves $\mathrm{ScS}_{2}$ and $\mathrm{ScS}_{3}$ are well simulated by the corrected earth-model. 

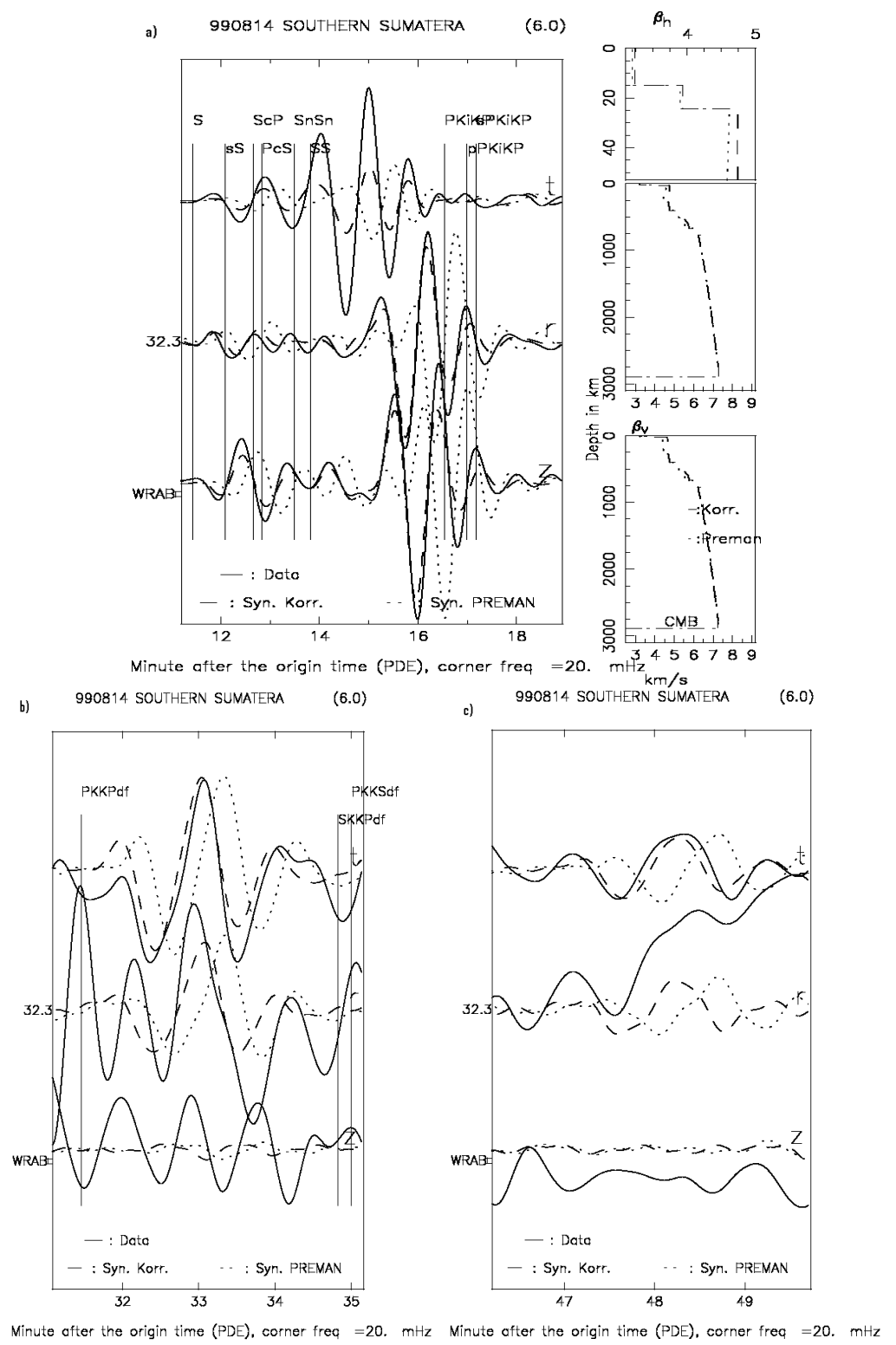

Figure 7 Seismogram Comparison of the earthquake C081499A, South Sumatra in the WRAB station: a. the S, Love and Rayleigh waves; b. $\mathrm{ScS}_{2} ; \mathrm{ScS}_{3}$.

The seismogram comparison of the earthquake B020399B, Sunda Straits in the WRAB Station, is shown in Figure 8. The earthquake B020399B and C081499A lie close to each other, and the PREMAN earth model gives the synthetic seismogram that arrives later than the measured ones, on all phases of $\mathrm{S}$ waves and their repetition depth waves, as well as on the surface waves Love 
and Rayleigh. The corrected model of the earth, in which the correction has a positive value for all layers of mantle, gives a synthetic seismogram that well approaches the measured seismogram from the S, SS, and Love and Rayleigh waves to the depth waves of $\mathrm{ScS}_{2}$ and $\mathrm{ScS}_{3}$.
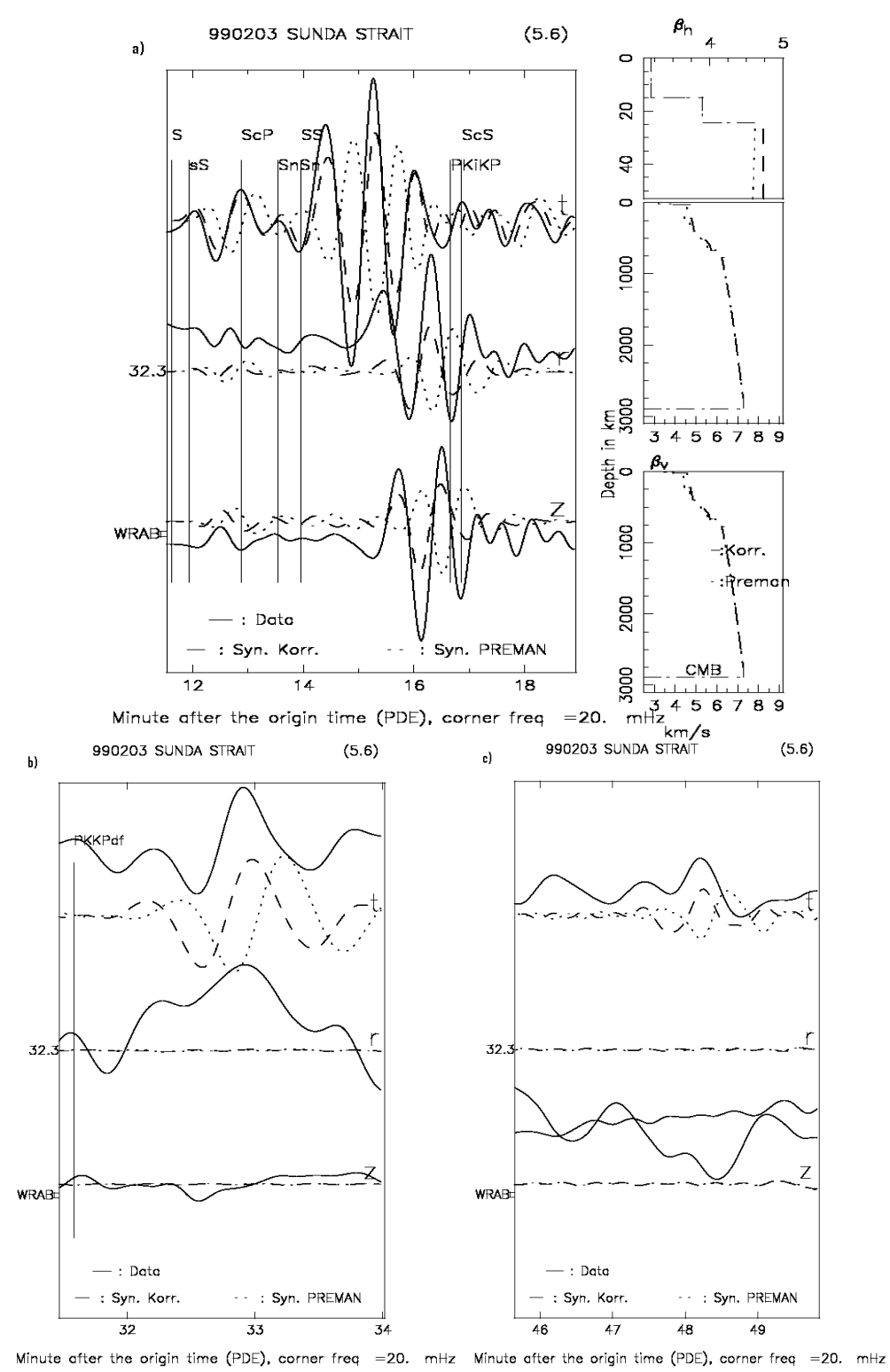

Figure 8 The seismogram fitting of the earthquake B020399B, Sunda Straits in The WRAB station: a. S, Love and Rayleigh wave; b. $\mathrm{ScS}_{2}$; and $\mathrm{ScS}_{3}$. 


\subsection{Discussion}

The interpretation of the earth structure using the travel time data set neglects the vertical anisotropic nature in the velocity of the wave travel. This is due to the use of the block structure, in which one point on one block can accommodate just one velocity value. Meanwhile, to accommodate the anisotropic nature we need the accommodating feature of more than one value. A description of the earth model with the spherical harmonic function can not describe the subduction structure. With this method, the data used to analyze the seismogram is subjected to a low-pass filter with a corner frequency of about 4 $\mathrm{mHz}$.

Tabel 2 The velocity structure of S wave between the PREMAN earth-model and the corrected model of the earth for the earthquake C103194B in the WRAB station; the zero-order coefficient and, on the upper mantle (6151 - 6346,6 km), the velocity gradient $\beta$ is also shown.

\begin{tabular}{cccc|ccc}
\hline \multicolumn{5}{c|}{ PREMAN } & \multicolumn{3}{c}{ C103194B - WRAB } \\
\hline $\begin{array}{c}\text { Radius } \\
(\mathbf{k m})\end{array}$ & $\begin{array}{c}\boldsymbol{\beta}_{\mathbf{v}} \\
\mathbf{( k m} / \mathbf{s})\end{array}$ & $\begin{array}{c}\boldsymbol{\beta}_{\mathbf{h}} \\
\mathbf{( k m} / \mathbf{s})\end{array}$ & $\eta$ & $\begin{array}{c}\boldsymbol{\beta}_{\mathbf{v}} \\
\mathbf{k m} / \mathbf{s})\end{array}$ & $\begin{array}{c}\boldsymbol{\beta}_{\mathbf{h}} \\
\mathbf{( k m} / \mathbf{s})\end{array}$ & $\eta$ \\
\hline 3480 & 6,9254 & 6,9254 & 1,0 & 6,8864 & 6,9254 & 0,9943 \\
3630 & 11,1671 & 11,1671 & 1,0 & 11,1601 & 11,2071 & 0,9958 \\
5600 & 22,3459 & 22,3459 & 1,0 & 22,6159 & 22,6459 & 0,9986 \\
5701 & 9,9839 & 9,9839 & 1,0 & 10,2639 & 10,2839 & 0,9980 \\
5771 & 22,3512 & 22,3512 & 1,0 & 22,6312 & 22,5712 & 1,0026 \\
5971 & 8,9496 & 8,9496 & 1,0 & 9,2196 & 9,1496 & 1,0076 \\
6151 & 5,8582 & $-1,0839$ & 3,3687 & 5,7982 & 6,1569 & 0,9421 \\
& $-1,4678$ & 5,7176 & $-2,4778$ & $-1,4678$ & $-1,4278$ & 1,0280 \\
6291 & 5,8582 & $-1,0839$ & 3,3687 & 5,7982 & 6,1569 & 0,9421 \\
& $-1,4678$ & 5,7176 & $-2,4778$ & $-1,4678$ & $-1,4278$ & 1,0280 \\
6346,6 & 3,9000 & 3,9000 & 1,0 & 3,8900 & 3,9500 & 0.9848 \\
6356 & 3,2000 & 3,2000 & 1,0 & 3,1900 & 3,2500 & 0,9815 \\
6371 & & & & & & \\
\hline
\end{tabular}

This research analyzed the seismogram with a corner frequency of $20 \mathrm{mHz}$. On this corner frequency, we found a very great deviation between the measured seismogram and synthetic seismogram that is constructed from the PREMAN standard earth model. The PREMAN describes the feature of vertical isotropic in the velocity structure of the wave just on the upper layer of mantle. The 
anisotropic feature on the upper mantle was obtained through an analysis on the seismogram with the mode summation method.

Table 2 presents an example of the S-wave velocity on various earth mantle layers, in which the PREMAN earth-model was compared with the corrected model of the earth between the earthquake hypocenter C103194B and the WRAB observation station. The velocity structure of S-wave on the other earthquake can be seen by noticing the small box that contains the velocity structure of the S-wave that lies on the right side of Figures $2-8$. We can see that the vertical anisotropic ( $\eta$ value) occurs on all of the mantle layers, not only on the upper mantle layer as stated in the PREMAN earth-model. We notice further by comparing the second and third columns with each of the fifth and the sixth column that generally has positive anomaly on the velocity structure of S-wave occurred on all layers of mantle.

After the correction on the subduction structure under Java-Sumatra we found that the velocity structure of $\mathrm{S}$ wave should be corrected with the positive values down to the lowest layer of mantle i.e. CMB $(3480 \mathrm{~km})$. These corrections indicate that the features of vertical anisotropic are possessed by all earth mantle layers. The correctness of all magnitude of the correction should be ensured by analyzing the depth waves, in which the travel of this wave passes by all mantle-layers many times. This research analyzes the depth wave at a small epicentral-distance station, in which this enables us to investigate the mantle structures near the earth core. It is different from the yielded from traveltime based research of seismogram, in which they need data of the observation on stations with a great epicentral distance. The 1-D earth structure produced in this research could be used as a preliminary model for the tomography inversion, for the further research.

The wave propagation from the hypocenter of earthquakes in Sumatra to the WRAB observation station passes the subduction structure and the mantle-layer pressed by the movement of the Australia continent toward the north. The research result with a waveform analysis on S, SS , the Love and Rayleigh surface waves, and the depth waves $\mathrm{ScS}_{2}$ and $\mathrm{ScS}_{3}$ indicate that the pressed mantle-layer due to the tectonic process have a positive $\mathrm{S}$ wave velocity anomaly and the vertical anisotropic on all mantle-layers.

The analysis of the Love waveform gives us an opportunity to investigate the thickness of the earth crust although the corner frequency used is only $20 \mathrm{mHz}$. It is possible because the Love wave turns out to be very sensitive to the thickness of the earth crusts. This factor has been avoided by other seismologists, in which they analyze the earth-crust thickness with the data of dispersion and of the refraction travel time [14]. 


\section{$4 \quad$ Conclusion}

The velocity structure of S wave on the subduction structure under SumatraJava has been investigated by the seismogram analysis in the time domain and the three Cartesian components simultaneously. The analyzed earthquakes lie in Sumatra and the observation station is in WRAB, North Australia. The data set used in this research makes use of all data points contained in the seismogram, unlike the data set of the travel time, in which only a small part of the data points are contained in the seismogram. The synthetic seismogram is calculated with the GEMINI methods, that is equivalent to the Mode Summation method. To simplify the waveform, the seismograms are low-pass filtered, with the corner frequency set on $20 \mathrm{mHz}$.

The seismogram comparison indicates that the synthetic seismogram of the earthquakes in Sumatra recorded in WRAB, North Australia, was calculated by the PREMAN preliminary model of the earth indicates lagged arrival-times than its counterpart measured wave phase, on all kinds of waves. Corrections are imposed on gradient $\beta_{\mathrm{h}}$, changed into positive and the zero order coefficients on the polynomial function of the $\mathrm{S}$ wave velocity on every mantle-layer gave a positive correction. The correction has different values for $\beta_{\mathrm{h}}$ and $\beta_{\mathrm{v}}$; this enables the vertical isotropic to occur in the mantle layers below the upper mantle. Good fittings were obtained on the surface waves of Love and Rayleigh, the shallow mantle waves S and SS, and the repetitive depth waves of $\mathrm{ScS}_{2}$ and $\mathrm{ScS}_{3}$.

The result of $\mathrm{S}$ wave velocity from the analysis on the seismogram of the earthquakes in Sumatra recorded in WRAB indicates that the positive correction occurs on all mantle-layers. This correction validity is also proved by the fitting in the repetitive depth waves. It states that the part of mantle that experiences a press due to the tectonic process (the Australia plate moves toward the north) has an anomaly of positive S velocity and the vertical anisotropic on all mantlelayers.

\section{Acknowledgement}

Gratitude is addressed to Dr. Dalkomo and Prof. Friedrich that has developed the GEMINI program with me, as well as to Prof. Wielandt. Gratitude is also addressed to IRIS (International Seismogram Network) that has provided the seismogram data for this research and its complementary software. The main program was written with the non-commercial software Intel FORTRAN, and the pictures in this paper are written with the PGPLOT and GMT software. This research was funded by Dana Dikti (High Education Fund) number 019SP3PPDP2MII2006. 


\section{Reference}

[1] Vasco, D., Johnson, L., Pulliam, R., Lateral variations in mantle velocity structure and discontinuities determined from $P, P P, S, S S$, and SS-ScS travel time residuals, J. Geophys. Res., 100, 24037-24059, 1995.

[2] Zhou, H., A high-resolution P wave model for the top $1200 \mathrm{~km}$ of the mantle, J. Geophys. Res., 101, 27791-27810, 1996.

[3] Grand, S., van der Hilst, R., Widiyantoro, S., Global seismic tomography: a snapshot of convection in the Earth, GSA Today, 7, 1-7, 1997.

[4] van der Hilst, R., Widiyantoro, S., Engdahl, E., Evidence for deep mantle circulation from global tomography, Nature, 386, 578-584, 1997.

[5] Bijwaard, H., Spakman, W., Engdahl, E., Closing the gap between regional and global travel time tomography, J. Geophys. Res., 103, 30055-30078, 1998.

[6] Boschi, L., Dziewonski, A., High- and low-resolution images of the Earth's mantle: Implications of different approaches to tomographic modeling, J. Geophys. Res., 104, 25567-25594, 1999.

[7] Zhao, D., Seismic structure and origin of hotspots and mantle plumes. Earth Planet, Sci. Lett., 192, 251-265, 2001.

[8] Zhao, D., Global tomographic images of mantle plumes and subducting slabs: insight into deep Earth dynamics, Physics of the Earth and Planetary Interiors, 146, 3-34, 2004.

[9] Lay, T., Williams, Q., Garnero, E., The core-mantle boundary layer and deep Earth dynamics, Nature, 392, 461-468, 1998.

[10] Garnero, E., Heterogeneity of the lowermost mantle, Annu. Rev. Earth Planet. Sci., 28, 509-537, 2000.

[11] Nataf, H., Seismic imaging of mantle plumes, Annu. Rev. Earth Planet. Sci., 28, 391-417, 2000.

[12] Dziewonski, A., Mapping the lower mantle: determination of lateral heterogeneity in P velocity up to degree and order 6, J. Geophys. Res., 89, 5929-5952, 1984.

[13] Su, W., Woodward, R., Dziewonski, A., Degree 12 model of shear velocity heterogeneity in the mantle, J. Geophys. Res., 99, 6945-6980, 1994.

[14] Mooney, W., Laske, G., Master, G., CRUST 5.1: a global crustal model at $5^{\circ} \times 5^{\circ}$, J. Geophys. Res., 103, 727-747, 1998.

[15] Flanagan, M., Shearer, P., Global mapping of topography on transition zone velocity discontinuities by stacking SS precursors, J. Geophys. Res., 103, 2673-2692, 1998.

[16] Engdahl, E., van der Hilst, R., Buland, R., Global teleseismic earthquake relocation with improved travel times and procedures for depth determination, Bull. Seismol. Soc. Am., 88, 722-743, 1998. 
[17] Bulland, R. \& Chapman, C., Travel time Calculation, BSSA, 73, 12711302, 1983.

[18] Dalkolmo, J., Synthetische Seismogramme fuer eine sphaerisch symmetrische, nichtrotierend Erde durch direkte Berechnung der Greenschen Funktion, Diplomarbeit, Inst. fuer Geophys., Uni. Stuttgart, 1993.

[19] Friederich, W. \& Dalkolmo, J., Complete synthetic seismograms for a spherically symmetric earth by a numerical computation of the green's function in the frequency domain, Geophys. J. Int., 122, 537-550, 1995.

[20] Dziewonski, A.M. \& Anderson, D.L., Preliminary reference Earth model, Phys. of the Earth and Plan. Int., 25, 297 - 356, 1981.

[21] Wysession, M., Lay, T., Revenaugh, J., The D" discontinuity and its implications, In: Gurnis, M., Buffett, B., Knittle, K., Wysession, M. (Eds.), The Core-Mantle Boundary. AGU, pp. 273-297, 1998.

[22] L. Boschi, A.M. Dziewonski, Whole Earth tomography from delay times of $P, P c P$, and PKP phases: lateral heterogeneities in the outer core or radial anisotropy in the mantle?, J. Geophys. Res., 105, 13675-13696, 2000. 